\title{
Antigenicity of borrelial protein BBK32 fragments in early Lyme borreliosis
}

Correspondence

Pekka Lahdenne

pekka.lahdenne@hus.fi

Received 10 March 2006

Accepted 8 August 2006

\author{
Pekka Lahdenne, ${ }^{1}$ Heikki Sarvas, ${ }^{2,3}$ Riikka Kajanus, ${ }^{2}$ Miia Eholuoto, ${ }^{2}$ \\ Heidi Sillanpää ${ }^{2}$ and Ilkka Seppälä 2,3
${ }^{1}$ Hospital for Children and Adolescents, University of Helsinki, Stenbäckinkatu 11, FIN-00290 Helsinki, Finland \\ ${ }^{2}$ Department of Bacteriology and Immunology, Haartman Institute, University of Helsinki, \\ Haartmaninkatu 3, FIN-00014 Helsinki, Finland \\ ${ }^{3}$ HUSLAB Laboratory Diagnostics, Helsinki University Central Hospital, Haartmaninkatu 3, \\ FIN-00290 Helsinki, Finland
}

\begin{abstract}
Recombinantly produced borrelial BBK32 protein fragments originating from Borrelia burgdorferi sensu stricto, Borrelia garinii and Borrelia afzelii were evaluated as antigens in the serology of Lyme borreliosis (LB). In ELISA, a mid-portion hydrophilic fragment reacted with LB patient sera. Of the 23 patients with culture- or PCR-positive erythema migrans (EM), $43 \%$ at diagnosis and $52 \%$ at convalescence were positive for at least one Borrelia species-specific variant BBK32 fragment antigen. In parallel ELISAs with BBK32 whole proteins from the three borrelial subspecies as antigens, $17 \%$ at diagnosis and $26 \%$ at convalescence were positive. These results suggest that BBK32 protein fragments may improve the early lgG serology of LB compared to the BBK32 whole protein.
\end{abstract}

\section{INTRODUCTION}

Laboratory verification of Lyme borreliosis (LB) relies mainly on serology, because culture of the causative agent from clinical samples or PCR-based methods seem to be too insensitive and therefore unfeasible in clinical practice (Nadelman \& Wormser, 1998; Sigal, 1998; Wormser et al., 1998). For serologic confirmation of Borrelia burgdorferi infection, a two-step approach with ELISA as a screening test and Western blotting as a confirmatory test is recommended by the Centers for Disease Control (CDC) (Brown et al., 1999). This approach has been challenged not only because it is labour-intensive and time-consuming, but also because the criteria for a positive Western blot vary according to which Borrelia strains are used as antigens and to local variations in the infective species of Borrelia (Robertson et al., 2000). In Europe and Asia especially, the development of a uniform approach for the serological evaluation of LB is complicated by the prevalence of organisms from the three or more genospecies of B. burgdorferi sensu lato and by significant antigenic variation between and within each genospecies (Wang et al., 1999). Moreover, in the USA 'Borrelia lonestari' exposure may confound serological results (Stromdahl et al., 2003).

Abbreviations: ACA, acrodermatitis chronica atrophicans; ASO, antistreptolysin; CDC, Centers for Disease Control; EM, erythema migrans; LA, Lyme arthritis; LB, Lyme borreliosis; NB, neuroborreliosis; RF, rheumatoid factor.
A contemporary approach to improve the performance of serological assays has been to use borrelial peptides or recombinant proteins as antigens (Liang et al., 1999; Magnarelli et al., 2000; Schulte-Spechtel et al., 2003). Despite the improvements in ELISAs, they still have the drawback of lacking sensitivity for early disease, although a few recent studies have suggested that borrelial VlsE protein or a conserved peptide from this protein, IR6, might be useful antigens for laboratory diagnosis of early LB (Magnarelli et al., 2002; Schulte-Spechtel et al., 2003; Marangoni et al., 2005, Goettner et al., 2005). We have shown previously that recombinantly produced borrelial BBK32 proteins might be useful as serodiagnostic antigens in LB (Heikkilä et al., 2002a; Lahdenne et al., 2003). In the serology of erythema migrans (EM), the early skin lesion of $\mathrm{LB}$, recent results have indicated that a BBK32 IgG, but not IgM, ELISA might exceed the performance of routinely used serological assays that are based on flagellin and/or borrelial whole-cell lysate antigens (Lahdenne et al., 2003).

Differentiation between infected and non-infected persons with the BBK32 protein antigens is, however, often suboptimal, because in diagnostic enzyme immunoassays, non-specific background signals may cause problems (Heikkilä et al., 2002a; Lahdenne et al., 2003). In the present study, to increase specificity we designed shorter fragments of the borrelial BBK32 proteins and evaluated these recombinant proteins as antigens in the IgG serology of early LB. 


\section{METHODS}

Bacterial strains. Finnish borrelial strains were received from the National Public Health Institute, Turku, Finland. B. burgdorferi sensu stricto (hereafter referred to as B. burgdorferi) strain Ia was isolated from the cerebrospinal fluid of a Finnish patient with neuroborreliosis (NB). Borrelia afzelii strain A91 and Borrelia garinii strain 40 were isolated from skin biopsy samples of Finnish patients with EM (Heikkilä et al., 2002b). The genotypes of the cultured borreliae were confirmed by sequencing a fragment of the flagellin gene (Junttila et al., 1999). Escherichia coli strains TOP10 (Invitrogen) and BL21 (Amersham Pharmacia Biotech) were used as host cells for cloning and expression of recombinant proteins.

Borreliae culture and DNA isolation. Borreliae were cultivated in BSK-H (Barbour-Stoenner-Kelly) medium (Sigma) with $5 \%$ $\mathrm{CO}_{2}$ at $33^{\circ} \mathrm{C}$. Borrelial genomic DNA was purified with a Dneasy Tissue Kit (Qiagen). Purified DNA was used in PCR and in cloning experiments.

Cloning and expression of BBK32 protein fragments. Cloning and expression of BBK32 whole proteins has been described previously (Heikkilä et al., 2002a). BBK32 whole proteins consist of 352-360 aa and the predicted molecular masses are around $38 \mathrm{kDa}$ (Heikkilä et al., 2002a). DNA and protein sequences were analysed with Lasergene software (DNASTAR). Expression primers were designed for N-terminal (Fragment 1) and mid-portion (Fragment 2) fragments of the putative mature BBK32 protein (Table 1 ). The sequences were selected from areas of predicted hydrophilicity (Fig. 1). For each borrelial strain, the $b b k 32$ fragment sequences were generated by PCR amplification of B. burgdorferi genomic DNA, as described previously (Heikkilä et al., 2002a). For expression of the $b b k 32$ fragments, glutathione $S$-transferase (GST) fusion protein constructs were generated. Hyperexpression of recombinant proteins was generated according to the manufacturer's instructions
(Amersham Pharmacia Biotech). Purification was done with Glutathione Sepharose 4B columns. The expression and purity of the fusion proteins was confirmed by SDS-PAGE.

ELISA. ELISA for BBK32 whole-protein antibodies has been described previously (Heikkilä et al., 2002a). For ELISAs measuring IgG antibodies to BBK32 protein fragments, the wells in a microtitre plate (Greiner) were coated with $50 \mathrm{ng}$ of the recombinant BBK32 fragment proteins overnight at $4{ }^{\circ} \mathrm{C}$. After washing, post-coating was done with $1 \%$ bovine serum albumin (BSA; Sigma) for $2 \mathrm{~h}$ at $37^{\circ} \mathrm{C}$ with or without an in-house $2 \%$ E. coli lysate (XL-1 Blue MRF'; Stratagene). Serum samples were diluted $1: 100$ in $5 \mathrm{mg} \mathrm{BSA} \mathrm{ml}^{-1}$ / $0 \cdot 1 \mathrm{M} \mathrm{NaCl} / 0 \cdot 04 \%$ Tween 20 buffer (BSA-NaCl-Tween). Samples $(100 \mu \mathrm{l})$ of $1: 100$ diluted serum were added to the wells and incubated overnight at $4{ }^{\circ} \mathrm{C}$. After washing, the wells were incubated with alkaline phosphatase-conjugated rabbit anti-human IgG (Jackson Immuno Research Laboratories) 1:5000 in BSA-NaClTween for $2 \mathrm{~h}$ at room temperature. The reactions were visualized with $1 \mathrm{mg}$ 4-nitrophenylphosphate $\mathrm{ml}^{-1}$ (Boehringer Mannheim) in diethanolamine buffer, $\mathrm{pH} 10 \cdot 0 . \mathrm{OD}_{405}$ measurements were taken after 10 min using an iEMS Reader MF (Thermo Labsystems). The cut-off for the ELISAs was defined as the mean +2 SD of values for healthy blood donors.

As control assays, flagellum IgM and IgG ELISAs were performed with commercially available ELISA kits (Dako) with modifications (Seppälä et al., 1994) and with a new recombinant protein ELISA kit (Biomedica) containing borrelial recombinant proteins OspC, p18, p100 and VlsE. The cut-off for positivity was determined according to the manufacturer's instructions in the kit.

Patient samples. Human serum samples were collected from 23 patients in Finland with culture- or PCR-positive EM, six patients with NB, seven with Lyme arthritis (LA) and three patients with acrodermatitis chronica atrophicans (ACA). Samples were collected from EM patients at diagnosis (acute) and 1-3 months after

Table 1. Sequences and locations of the expression primers for the BBK32 protein fragments (Fragment 1 and Fragment 2) from B. afzelii A91, B. burgdorferi la or B. garinii 40

\begin{tabular}{|c|c|c|c|}
\hline Species & Primer $\left(5^{\prime}-3^{\prime}\right)^{*}$ & Location (bp) & Source $\dagger$ \\
\hline \multirow[t]{6}{*}{ B. afzelii } & Fragment 1 (amino acid range $20-126$ ) & & \\
\hline & CCGGATCCGATTTATTCATAAGAGATGAAAT & $58-80$ & AF472525 \\
\hline & CCCTCGAGTTTATGCTCATCTTGCTGTTT & $360-378$ & \\
\hline & Fragment 2 (amino acid range $130-220)$ & & \\
\hline & CCGGATCCCAAGGAAGTTTAAGTTTTCTTA & $388-406$ & \\
\hline & CCCTCGAGTGAACTTACATTATATTTAACAC & $642-660$ & \\
\hline \multirow[t]{6}{*}{ B. burgdorferi } & Fragment 1 (amino acid range $21-127$ ) & & \\
\hline & CGCGGATCCGATTTATTCATAAGATATGAAATG & $61-84$ & AF472532 \\
\hline & CCGCTCGAGATCATTTATATTCATCTTGTTGTTTTTG & $358-381$ & \\
\hline & Fragment 2 (amino acid range $131-228)$ & & \\
\hline & CGCGGATCCCAAGGAAGTTTAAATTCCCTTA & $391-412$ & \\
\hline & CCGCTCGAGATCATGAATCTACATTATATTTAACACC & $661-684$ & \\
\hline \multirow[t]{6}{*}{ B. garinii } & Fragment 1 (amino acid range $21-127$ ) & & \\
\hline & CGCGGATCCGATTTATTCATAAGATATGAAATG & $61-84$ & AF472529 \\
\hline & CCGCTCGAGATCATTTATATTCATCTTGTTGTTTTTG & $358-381$ & \\
\hline & Fragment 2 (amino acid range $131-228$ ) & & \\
\hline & CGCGGATCCAAAGGAAGTTTAAATTCCCTTA & $391-412$ & \\
\hline & CCGCTCGAGATCATGAATCTACATTATATTTAACACC & $661-684$ & \\
\hline
\end{tabular}

${ }^{\star}$ Underlined sequences are restriction enzyme sites.

$\uparrow$ NCBI accession number. 


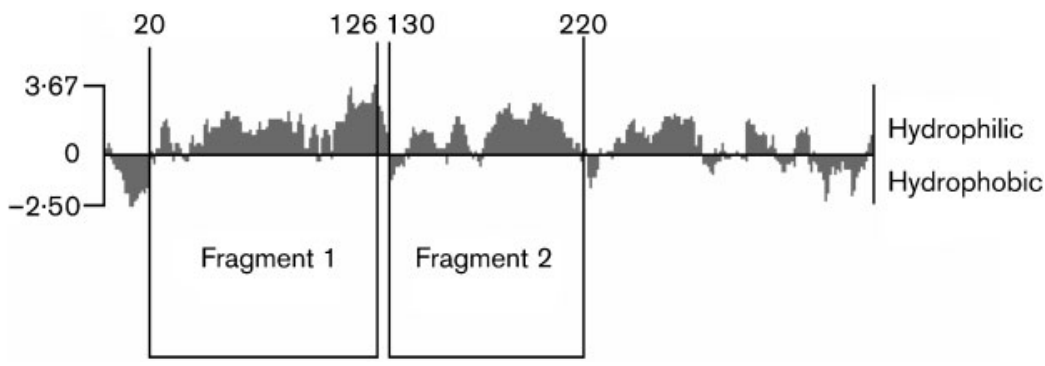

Fig. 1. Hydrophilicity plot (Lasergene; DNASTAR) and amino acid localization of designed protein fragments of the borrelial BBK32 protein from $B$. afzelii A91.

treatment (convalescent). Culture and/or PCR of the skin biopsies showed that of the 23 patients with EM, 17 were infected by B. afzelii. In the patients with disseminated $\mathrm{LB}$, the clinical manifestations agreed with the CDC criteria for LB (Wharton et al., 1990). The clinical diagnosis was confirmed in ELISA by demonstrating antibodies against flagellin (serum and cerebrospinal fluid samples) and $B$. burgdorferi whole-cell lysate antigen (serum samples). As controls we used serum samples from patients with syphilis $(n=5)$, rheumatoid factor (RF) positivity $(n=5)$, anti-streptolysin (ASO) positivity $(n=5)$, high Salmonella $(n=5)$ or Yersinia enterocolitica $(n=5)$ antibody titres, or from healthy blood donors.

Statistical analyses. Microsoft Excel 2000 and GraphPad PRISM 3.0 were used for calculations of standard statistics.

\section{RESULTS AND DISCUSSION}

\section{Antigenicity of the BBK32 fragments}

Sera from three patients previously known for being highly positive for $\mathrm{LB}$ (patients with $\mathrm{NB}$ ) were used for a preliminary evaluation of the antigenicity of BBK32 fragments. Fragment 2 was superior to the Fragment 1 in differentiating between patient and control samples (data not shown). Post-coating of the wells with $1 \%$ BSA decreased background signals in ELISA, but adding $2 \% E$. coli lysate did not improve the assay. The signals with Fragment 1 did not differ from those with the control antigen (GST). Consequently, Fragment 1 was not studied further.

\section{Analysis of the amino acid sequence of BBK32 Fragment 2}

Fragments 2 from B. garinii and B. burgdorferi consisted of 98 residues and differed by only one amino acid. There was a deletion of 7 residues in Fragment 2 of B. afzelii. In addition, 16 residues differed from the $B$. garinii and B. burgdorferi BBK32 sequences. A GenBank BLAST search showed that all sequences homologous to BBK32 Fragment 2 were associated with borreliae (data not shown).

\section{BBK32 protein fragments as antigens in the serology of LB}

Of the 23 European patients with EM, 10 (43\%) at diagnosis or $12(52 \%)$ at convalescence were positive with at least one variant of the Fragment 2 antigens (Table 2). All patients with positive BBK32 antibodies had been infected with $B$. afzelii. In parallel ELISAs with BBK32 whole proteins from the three borrelial subspecies as antigens, 3-4 (13-17\%) at diagnosis and 2-6 (9-26\%) at convalescence were positive (Table 2, Fig. 2). Of the Fragment 2 antigens originating from the three borrelial subspecies, the positivity rate was highest with those from B. afzelii (Fig. 2). The same species

Table 2. Positivity rate (\%) of IgG antibodies to the three borrelial BBK32 protein fragments (Fragm2) and BBK32 whole-protein antigens (BBK32) from B. afzelii (B.afz), B. garinii (B.gar) and B. burgdorferi (B. bur)

Percentages of positive antibodies are also given for commercial flagellum antigens (Fla-IgM, Fla-IgG; Dako) and a commercial combination of recombinant proteins (Recomb. IgG; Biomedica). Serum samples were from patients with EM at diagnosis (EM I) and at convalescence (EM II), and from patients with disseminated borreliosis (LB) (including NB, LA, ACA). Control samples were from patients with syphilis, RF positivity, ASO positivity and high Salmonella or Yersinia enterocolitica antibody titres, and from healthy blood donors (BD).

\begin{tabular}{|c|c|c|c|c|c|c|c|c|c|c|c|}
\hline Patient sample $(n)$ & \multicolumn{11}{|c|}{ Antigen } \\
\hline EM I (23) & 43 & 22 & 22 & 43 & 17 & 13 & 13 & 17 & 30 & 22 & $50^{*}$ \\
\hline EM II (23) & 52 & 17 & 13 & 52 & 26 & 9 & 13 & 26 & 26 & 22 & $55^{*}$ \\
\hline LB (16) & 94 & 94 & 94 & 94 & 100 & 88 & 94 & 100 & 31 & 100 & 100 \\
\hline
\end{tabular}

${ }^{\star}$ Twenty-two samples studied. 

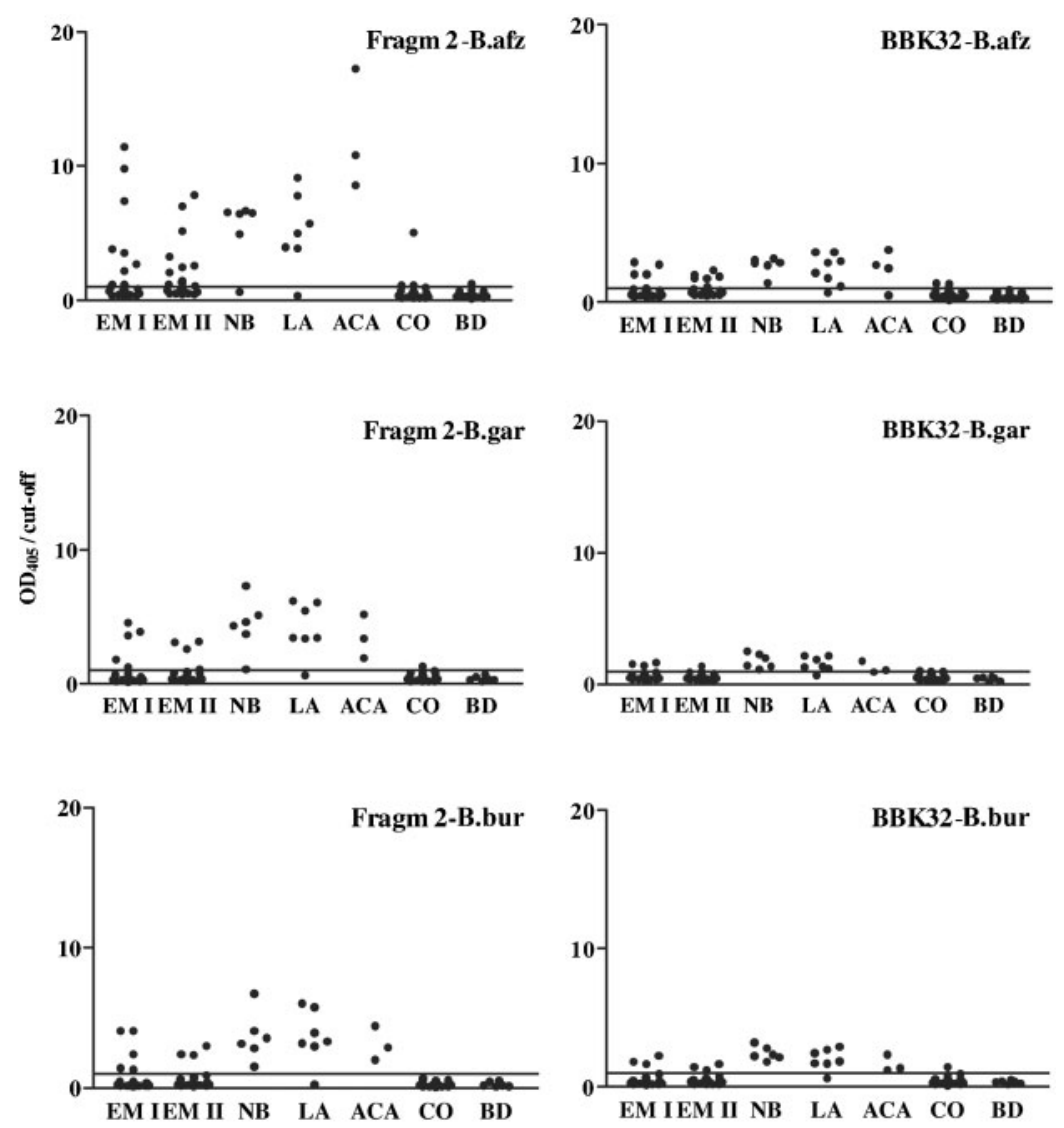

Fig. 2. ELISA $\mathrm{OD}_{405} /$ cut-off values of patients with EM (EM I at diagnosis and EM II at convalescence), NB, LA and ACA. IgG antibodies to the recombinant BBK32 protein mid-portion fragment (Fragm 2) and the BBK32 whole protein (BBK32) from B. afzelii (B.afz), B. garinii (B.gar) and B. burgdorferi (B.bur) were measured. Control samples (CO) were from 25 patients with syphilis, RF positivity, ASO positivity, high Salmonella or Yersinia enterocolitica antibody titres, and from 6-46 healthy blood donors (BD). The level of positivity $\left(\mathrm{OD}_{405} /\right.$ cut-off $\left.>1\right)$ is indicated by a horizontal line.

pattern was observed for BBK32 whole proteins (Fig. 2). The cut-off levels based on the optical density values of healthy blood donors were $0 \cdot 3-0.5$ with Fragment 2 antigens compared to $1 \cdot 0-1 \cdot 3$ with $\mathrm{BBK} 32$ whole-protein antigens.

Of the disease controls, one patient with syphilis showed positive optical density levels with BBK32 Fragment 2 from B. afzelii and with BBK32 whole protein from the same genospecies. Two more control samples were marginally positive for BBK32 Fragment 2 or whole-protein antigens (Fig. 2, Table 2).

As control assays for LB serology, commercial flagellumbased IgM and IgG ELISAs and a commercial recombinant protein IgG ELISA were used. The results are shown in Table 2. Of the 25 disease controls, three patients with syphilis and one RF-positive patient showed IgG antiflagellum antibodies, and two patients with syphilis, two RFpositive patients, two patients positive for Salmonella antibodies and one patient positive for Yersinia antibodies were positive in the commercial recombinant protein IgG ELISA. All the positive control samples were low positives.

\section{Sensitivity and specificity of the serological assays}

In patients with EM at diagnosis, the combined sensitivity of the BBK32 IgG ELISA with Fragment 2 or BBK32 whole-protein antigens was 43 or $17 \%$, respectively. With the same patient samples, the sensitivities for flagellum IgM, flagellum IgG or the commercial recombinant protein IgG ELISAs were 30, 22 or $50 \%$, respectively. In disseminated disease, the sensitivities of BBK32 Fragment 2, BBK32 whole protein, flagellum IgM, flagellum IgG or commercial recombinant protein IgG ELISAs were 94, 100, 33, 100 or $100 \%$, respectively. The specificities of BBK32 Fragment 2, BBK32 whole protein, flagellum IgM, flagellum IgG or commercial recombinant protein IgG ELISAs calculated with the 25 disease control samples were $88,88,92,84$ or $72 \%$, respectively.

\section{Conclusions}

The present study provides evidence for antigenicity of recombinantly produced $\mathrm{BBK} 32$ borrelial proteins in the mid-portion, but not in the $\mathrm{N}$ terminus of the protein. The results suggest that the mid-portion hydrophilic protein fragment may improve the IgG serology of EM compared to the BBK32 whole-protein antigen. This improvement seemed to be mainly due to a decrease in non-specific background signals, leading to an enhanced operating range in the test and, thus, better differentiation between patient and control samples. The antigenic efficiency of the BBK32 fragment itself may not necessarily be better than that of the whole protein. In the serology for disseminated borreliosis, the performance of the BBK32 fragment and the 
whole protein as ELISA antigens did not seem to differ appreciably.

Recently, new serological assays containing several recombinantly produced borrelial proteins, originating from different pathogenic borrelial subspecies, have been introduced (Goettner et al., 2005). In the present study, the BBK32 ELISA was compared with one of these new ELISAs, containing a combination of recombinant proteins, and with a flagellumbased ELISA. In patients with EM, the sensitivities of the recombinant protein combination and the BBK32 protein fragment ELISAs were at the same level and exceeded that of the flagellum-based ELISA. However, the specificities of both commercial assays seemed to be lower than with the BBK32 antigen. In future studies, it will be interesting to see whether BBK32 could be combined with other recombinant borrelial proteins and whether this combination of antigens would increase the performance of the assay, either by specifying the immune reactivity or by increasing the sensitivity.

The antigenic mid-portion BBK32 protein fragment is in fact located in the $\mathrm{N}$-terminal domain of the protein, where the fibronectin binding site has also been mapped (Probert et al., 2001). Upon fibronectin binding, the BBK32 protein has recently been shown to undergo conformational changes (Kim et al., 2004), which theoretically might be unfavourable for the antibody-antigen interaction. Nevertheless, the mid-portion fragment of the protein we used in the present study showed better performance than the BBK32 whole protein. It can be speculated, though, that in further studies with the BBK32 antigen, the interaction with fibronectin should be taken into consideration.

The majority of the patients in the EM series were infected with $B$. afzelii which agrees with the best serologic performance from the corresponding homologous BBK32 protein fragment. It is possible that the sequence heterogeneity (up to $21 \%$ ) between the mid-portion protein fragments from $B$. afzelii compared to other genospecies would account for the discrepancies in the serology. In addition, there seem to be differences in the appearance and development of EM lesions between borrelial genospecies (Carlsson et al., 2003), which might also explain variant timing of antibody evolution. Future studies will be aimed at dissecting how BBK32 fragments from different borrelial genospecies would perform when testing serum samples from epidemiologically diverse regions.

In the present study, we focused on IgG serology because in our previous studies the sensitivity of IgM serology at an acceptable specificity level with BBK32 whole-protein antigens has been low in patients with early LB (Heikkilä et al., 2002a). When used to assess the first line of antibody response in LB, IgM serology is often prone to specificity problems due to cross-reactive antibodies from other infections (Brown et al., 1999).

In conclusion, BBK32 protein fragments seem to improve the sensitivity of the IgG serology of early LB at the same specificity level compared to the BBK32 whole protein. More research is needed to clarify the specific antigenic epitopes in BBK32 proteins of various borrelial subspecies. For further development of LB serology, the BBK32 antigen may be an interesting candidate for combination with other recombinant borrelial proteins. This study also underlines the fact that the heterogeneity of Lyme disease borreliae must be taken into consideration in the microbiological diagnosis of LB in European patients.

\section{ACKNOWLEDGEMENTS}

This study was supported by the National Technology Agency (TEKES), Finland, and the Helsinki Central Hospital Research Funds, Helsinki, Finland.

\section{REFERENCES}

Brown, S. L., Hansen, S. L. \& Langone, J. J. (1999). Role of serology in the diagnosis of Lyme disease. JAMA 282, 62-66.

Carlsson, S.-A., Granlund, H., Jansson, C., Nyman, D. \& Wahlberg, P. (2003). Characteristics of erythema migrans in Borrelia afzelii and Borrelia garinii infections. Scand J Infect Dis 35, 31-33.

Goettner, G., Schulte-Spechtel, U., Hillerman, R., Liegl, G., Wilske, B. \& Fingerle, V. (2005). Improvement of Lyme borreliosis serodiagnosis by a newly developed recombinant immunoglobulin G (IgG) and IgM line immunoblot assay and addition of VlsE and DbpA homologues. J Clin Microbiol 43, 3602-3609.

Heikkilä, T., Seppälä, I., Saxen, H., Panelius, J., Peltomaa, M., Julin, T., Carlsson, S. A. \& Lahdenne, P. (2002). Recombinant BBK32 protein in serodiagnosis of early and late Lyme borreliosis. J Clin Microbiol 40, 1174-1180.

Heikkilä, T., Seppälä, I., Saxen, H., Panelius, J., Yrjänäinen, H. \& Lahdenne, P. (2002). Species-specific serodiagnosis of Lyme arthritis and neuroborreliosis due to Borrelia burgdorferi sensu stricto, $B$. afzelii, and B. garinii by using decorin binding protein A. J Clin Microbiol 40, 453-460.

Junttila, J., Peltomaa, M., Soini, H., Marjamäki, M. \& Viljanen, M. K. (1999). Prevalence of Borrelia burgdorferi in Ixodes ricinus ticks in urban recreational areas of Helsinki. J Clin Microbiol 37, 1361-1365.

Kim, J. H., Singvall, J., Schward-Linek, U., Johnson, B. J. B., Potts, J. R. \& Höök, M. (2004). BBK32, a fibronectin binding MSCRAMM from Borrelia burgdorferi, contains a disordered region that undergoes a conformational change on ligand binding. J Biol Chem 279, 41706-41714.

Lahdenne, P., Panelius, J., Saxen, H., Heikkilä, T., Sillanpää, H., Peltomaa, M., Arnez, M., Huppertz, H.-I. \& Seppälä, I. J. (2003). Improved serodiagnosis of erythema migrans using novel recombinant borrelial BBK32 antigens. J Med Microbiol 52, 563-567.

Liang, F. T., Steere, A. C., Marques, A. R., Johnson, B. J. B., Miller, J. N. \& Philipp, M. T. (1999). Sensitive and specific serodiagnosis of Lyme disease by enzyme-linked immunosorbent assay with a peptide based on an immunodominant conserved region of Borrelia burgdorferi VlsE. J Clin Microbiol 37, 3990-3996.

Magnarelli, L. A., ljdo, J. W., Padula, S. J., Flavell, R. A. \& Fikrig, E. (2000). Serologic diagnosis of Lyme borreliosis by using enzymelinked immunosorbent assays with recombinant antigens. J Clin Microbiol 38, 1735-1739.

Magnarelli, L. A., Lawrenz, M., Norris, S. J. \& Fikrig, E. (2002). Comparative reactivity of human sera to recombinant VlsE and 
other Borrelia burgdorferi antigens in class-specific enzyme-linked immunosorbent assays for Lyme borreliosis. J Med Microbiol 51, 649-655.

Marangoni, A., Sparacino, M., Mondardini, V., Cavrini, F., Storni, E., Donati, M., Cevenini, R. \& Sambri, V. (2005). Comparative evaluation of two enzyme linked immunosorbent assay methods and three Western Blot methods for the diagnosis of culture-confirmed early Lyme borreliosis in Italy. New Microbiol 28, 37-43.

Nadelman, R. B. \& Wormser, G. P. (1998). Lyme borreliosis. Lancet 352, 557-565.

Probert, W. S., Kim, J. H., Höök, M. \& Johnson, B. J. B. (2001). Mapping the ligand-binding region of Borrelia burgdorferi fibronectin-binding protein BBK32. Infect Immun 69, 4129-4133.

Robertson, J., Guy, E., Andrews, N. \& 9 other authors (2000). A European multicenter study of immunoblotting in serodiagnosis of Lyme borreliosis. J Clin Microbiol 38, 2097-2102.

Schulte-Spechtel, U., Lehnert, G., Liegl, G., Fingerle, V., Heimerl, C., Johnson, B. J. B. \& Wilske, B. (2003). Significant improvement of the recombinant Borrelia-specific immunoglobulin G immunoblot test by addition of VlsE and a DbpA homologue derived from Borrelia garinii for diagnosis of early neuroborreliosis. J Clin Microbiol 41, 1299-1303.
Seppälä, I. J. T., Kroneld, R., Schauman, K., Forsen, K. O. \& Lassenius, R. (1994). Diagnosis of Lyme borreliosis: non-specific serological reactions with Borrelia burgdorferi sonicate antigen caused by IgG2 antibodies. J Med Microbiol 40, 293-302.

Sigal, L. H. (1998). Pitfalls in the diagnosis and management of Lyme disease. Arthritis Rheum 41, 195-204.

Stromdahl, E. Y., Williamson, P. C., Kollars, T. M., Jr, Evans, S. R., Barry, R. K., Vince, M. A. \& Dobbs, N. A. (2003). Evidence of Borrelia lonestari DNA in Amblyomma americanum (Acari: Ixodidae) removed from humans. J Clin Microbiol 41, 5557-5562.

Wang, G., van Dam, A. P., Schwartz, I. \& Dankert, J. (1999). Molecular typing of Borrelia burgdorferi sensu lato: taxonomic, epidemiologic and clinical implications. Clin Microbiol Rev 12, 633-653.

Wharton, M., Chorba, T. L., Vogt, R. L., Morse, D. L. \& Buehler, J. W. (1990). Case definitions for public health surveillance. Morb Mortal Wkly Rep 39, 19-21.

Wormser, G. P., Nowakowski, J., Nadelman, R. B., Bittker, S., Cooper, D. \& Pavia, C. (1998). Improving the yield of blood cultures for patients with early Lyme disease. J Clin Microbiol 36, 296-298. 